\title{
Three solutions for a partial discrete Dirichlet boundary value problem with $p$-Laplacian
}

Shaohong Wang ${ }^{1,2}$ and Zhan Zhou ${ }^{1,2^{*}}$ (D)

\section{"Correspondence:}

zzhou0321@hotmail.com

'School of Mathematics and

Information Science, Guangzhou

University, Guangzhou, P.R. China

${ }^{2}$ Center for Applied Mathematics,

Guangzhou University, Guangzhou,

P.R. China

\begin{abstract}
By employing critical point theory, we investigate the existence of solutions to a boundary value problem for a $p$-Laplacian partial difference equation depending on a real parameter. To be specific, we give precise estimates of the parameter to guarantee that the considered problem possesses at least three solutions. Furthermore, based on a strong maximum principle, we show that two of the obtained solutions are positive under some suitable assumptions of the nonlinearity.
\end{abstract}

Keywords: Boundary value problem; Partial difference equation; Critical point theory; $p$-Laplacian

\section{Introduction}

Let $\mathbb{Z}$ and $\mathbb{R}$ denote the sets of integers and real numbers, respectively. Define $\mathbb{Z}(a, b)=$ $\{a, a+1, \ldots, b\}$ with $a \leq b$ for any $a, b \in \mathbb{Z}$.

Given positive integers $m$ and $n$, we consider the following partial discrete problem, $\operatorname{denoted}\left(S_{\lambda}^{f, q}\right)$ :

$$
\begin{aligned}
& -\Delta_{1}\left[\phi_{p}\left(\Delta_{1} x(i-1, j)\right)\right]-\Delta_{2}\left[\phi_{p}\left(\Delta_{2} x(i, j-1)\right)\right]+q(i, j) \phi_{p}(x(i, j))=\lambda f((i, j), x(i, j)), \\
& (i, j) \in \mathbb{Z}(1, m) \times \mathbb{Z}(1, n),
\end{aligned}
$$

with Dirichlet boundary conditions

$$
\begin{array}{ll}
x(i, 0)=x(i, n+1)=0, & i \in \mathbb{Z}(0, m+1), \\
x(0, j)=x(m+1, j)=0, & j \in \mathbb{Z}(0, n+1) .
\end{array}
$$

Here $\Delta_{1}$ and $\Delta_{2}$ denote the forward difference operators defined by $\Delta_{1} x(i, j)=x(i+1, j)-$ $x(i, j)$ and $\Delta_{2} x(i, j)=x(i, j+1)-x(i, j), \Delta_{1}^{2} x(i, j)=\Delta_{1}\left(\Delta_{1} x(i, j)\right)$ and $\Delta_{2}^{2} x(i, j)=\Delta_{2}\left(\Delta_{2} x(i, j)\right)$, $\phi_{p}$ denotes the $p$-Laplacian operator, that is, $\phi_{p}(s)=|s|^{p-2} s, p>1, q(i, j) \geq 0$ for all $(i, j) \in$ $\mathbb{Z}(1, m) \times \mathbb{Z}(1, n)$, and $f((i, j), \cdot) \in C(\mathbb{R}, \mathbb{R})$ for each $(i, j) \in \mathbb{Z}(1, m) \times \mathbb{Z}(1, n)$.

(c) The Author(s) 2021. This article is licensed under a Creative Commons Attribution 4.0 International License, which permits use, sharing, adaptation, distribution and reproduction in any medium or format, as long as you give appropriate credit to the original author(s) and the source, provide a link to the Creative Commons licence, and indicate if changes were made. The images or other third party material in this article are included in the article's Creative Commons licence, unless indicated otherwise in a credit line to the material. If material is not included in the article's Creative Commons licence and your intended use is not permitted by statutory regulation or exceeds the permitted use, you will need to obtain permission directly from the copyright holder. To view a copy of this licence, visit http://creativecommons.org/licenses/by/4.0/. 
It is well known that the critical point theory has been playing an important role in the study of differential equations. For example, Ji [1] considered the following problem:

$$
\left\{\begin{array}{l}
-\operatorname{div}\left(|\nabla u|^{p(x)-2} \nabla u\right)+|u|^{p(x)-2} u=\lambda f(x, u)+\mu g(x, u) \quad \text { in } \Omega \\
B u=0 \quad \text { on } \partial \Omega .
\end{array}\right.
$$

By using Theorem 2 in [2], the author obtained some new results on the existence of three solutions for problem (1.1) which give information on the localization of the interval of the parameter. In 2020, Papageorgiou and Scapellato [3] studied the problem

$$
\left\{\begin{array}{l}
-\operatorname{div}\left(|D u|^{p-2} D u\right)-\operatorname{div}(D u)=\lambda|u|^{p-2} u+f(z, u) \quad \text { in } \Omega \\
\left.u\right|_{\partial \Omega}=0
\end{array}\right.
$$

By applying critical point theory, the authors showed that when the parameter $\lambda>0$ is small, problem (1.2) admits at least seven nontrivial solutions including two positive solutions, two negative solutions and three nodal solutions.

As the discrete analogues of differential equations, during the past decades, the theory of difference equations has been also developed continuously due to its theoretical background and realistic significance. For instance, difference equations have been used extensively as discrete mathematical models describing real-life scenarios in electrical circuit analysis, economics, dynamical systems, physics, biology, etc. [4-7]. On the other hand, the existence and multiplicity of solutions for difference equations have been widely studied by many scholars. For instance, Stevic [8] investigated the problem

$$
z_{n+1}=\alpha z_{n}^{a} w_{n}^{b}, \quad w_{n+1}=\beta w_{n-1}^{c} z_{n}^{d}, \quad n \in \mathbb{N}_{0},
$$

and presented closed form formulas for well-defined complex-valued solutions to (1.3) under some suitable assumptions of the parameters and initial values. Furthermore, it must be pointed out that Guo and Yu [9] first applied the critical point theory to study the existence of periodic and subharmonic solutions for a second-order difference equation in 2003. Since then, the critical point theory has become a powerful tool to deal with the nonlinear discrete problems, and many excellent results were acquired, concerning periodic solutions [10-12], homoclinic solutions [13-23], heteroclinic solutions [24, 25], boundary value problems [26-34] and so on.

Note that the difference equations mentioned above involve only one discrete variable, while the difference equations with two or more discrete variables, so-called partial difference equations, are also very meaningful and investigated. Here we focus on the following several papers.

In 2010, Galewski and Orpel [35] considered the problem $\left(E_{\lambda}^{f}\right)$ :

$$
\left\{\begin{array}{l}
-\Delta_{1}^{2} u(i-1, j)-\Delta_{2}^{2} u(i, j-1)=\lambda f((i, j), u(i, j)), \quad(i, j) \in \mathbb{Z}(1, m) \times \mathbb{Z}(1, n), \\
u(i, 0)=u(i, n+1)=0, \quad i \in \mathbb{Z}(1, m), \\
u(0, j)=u(m+1, j)=0, \quad j \in \mathbb{Z}(1, n) .
\end{array}\right.
$$


Following some ideas from [36], the authors rewrote $\left(E_{\lambda}^{f}\right)$ as a nonlinear algebraic system and obtained the existence of at least one nontrivial solution by applying critical point theory and some monotonicity results.

Similarly, in 2015, Heidarkhani and Imbesi [37] established some sufficient conditions to ensure that problem $\left(E_{\lambda}^{f}\right)$ possesses at least three distinct solutions, respectively, by employing two different critical points theorems.

In 2016, by making use of critical point theory and the same techniques as [35, 37], Imbesi and Bisci [38] further studied the nonlinear algebraic system corresponding to problem $\left(E_{\lambda}^{f}\right)$ and acquired two types of results: the existence of either an unbounded sequence of solutions or a sequence of pairwise distinct non-zero solutions that converges to zero.

Lately, Du and Zhou [39] dealt with a class of partial discrete Dirichlet boundary value problem involving the $p$-Laplacian, namely, problem $\left(S_{\lambda}^{f, q}\right)$ when $q(i, j)=0$ for any $(i, j) \in$ $\mathbb{Z}(1, m) \times \mathbb{Z}(1, n)$. By establishing the variational framework associated with $\left(S_{\lambda}^{f, 0}\right)$ and exploiting critical point theory, a series of results were obtained.

Inspired by the above research results, the aim of this paper is to investigate the existence of multiple solutions to problem $\left(S_{\lambda}^{f, q}\right)$. Note that problem $\left(E_{\lambda}^{f}\right)$ mentioned above is a special case of $\left(S_{\lambda}^{f, q}\right)$ when $p=2$ and $q(i, j)=0$ for any $(i, j) \in \mathbb{Z}(1, m) \times \mathbb{Z}(1, n)$. Besides, different from the skills in [37] and the main tools used in their proof, in this paper we construct the variational structure for $\left(S_{\lambda}^{f, q}\right)$ and transform the existence of solutions for $\left(S_{\lambda}^{f, q}\right)$ into that of critical points of the corresponding variational functional. Based on another three critical point theorem, the existence results of at least three solutions are established. Furthermore, under appropriate hypotheses on the nonlinearity $f$, we verify that $\left(S_{\lambda}^{f, q}\right)$ admits at least two positive solutions by using a strong maximum principle.

First of all, we give the following lemma (see Theorem 2.1 of [40]), which is the main tool of this paper.

Lemma 1.1 Let $X$ be a separable and reflexive real Banach space. $\Phi: X \rightarrow \mathbb{R}$ is a nonnegative continuously Gâteaux differentiable and sequentially weakly lower semicontinuous functional whose Gâteaux derivative admits a continuous inverse on $X^{*} . J: X \rightarrow \mathbb{R}$ is a continuously Gâteaux differentiable functional whose Gâteaux derivative is compact. Assume that there exists $x_{0} \in X$ such that $\Phi\left(x_{0}\right)=J\left(x_{0}\right)=0$ and that

(i) $\lim _{\|x\| \rightarrow+\infty}[\Phi(x)-\lambda J(x)]=+\infty$ for all $\lambda \in[0,+\infty)$;

Further, assume that there are $r>0, x_{1} \in X$ such that

(ii) $r<\Phi\left(x_{1}\right)$;

(iii) $\sup _{x \in \bar{\Phi}^{-1}(-\infty, r)} w(x)<\frac{r}{r+\Phi\left(x_{1}\right)} J\left(x_{1}\right)$.

Then, for each

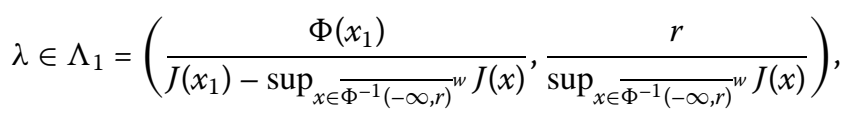

the equation

$$
\Phi^{\prime}(x)-\lambda J^{\prime}(x)=0
$$


has at least three solutions in $X$ and, moreover, for each $h>1$, there exist an open interval

$$
\Lambda_{2} \subseteq\left[0, \frac{h r}{\frac{r J\left(x_{1}\right)}{\Phi\left(x_{1}\right)}-\sup _{x \in \bar{\Phi}^{-1}(-\infty, r)} w(x)}\right]
$$

and a positive real number $\sigma$ such that, for each $\lambda \in \Lambda_{2}$, the equation (1.4) has at least three solutions in $X$ whose norms are less than $\sigma$.

The rest of this paper is organized as follows. In Sect. 2, we construct the variational structure for problem $\left(S_{\lambda}^{f, q}\right)$ and present a strong maximum principle as well as two useful inequalities. In Sect. 3, our main results are established. Furthermore, under suitable hypotheses on the nonlinearity $f$, two corollaries are obtained by employing the strong maximum principle. Finally, a concrete example is provided in Sect. 4 to illustrate our results.

\section{Preliminaries}

Consider the mn-dimensional Banach space

$$
\begin{aligned}
X= & \{x: \mathbb{Z}(0, m+1) \times \mathbb{Z}(0, n+1) \rightarrow \mathbb{R} \text { such that } x(i, 0)=x(i, n+1)=0, \\
& i \in \mathbb{Z}(0, m+1) \text { and } x(0, j)=x(m+1, j)=0, j \in \mathbb{Z}(0, n+1)\},
\end{aligned}
$$

endowed by the norm

$$
\|x\|=\left(\sum_{j=1}^{n} \sum_{i=1}^{m+1}\left|\Delta_{1} x(i-1, j)\right|^{p}+\sum_{i=1}^{m} \sum_{j=1}^{n+1}\left|\Delta_{2} x(i, j-1)\right|^{p}+\sum_{j=1}^{n} \sum_{i=1}^{m} q(i, j)|x(i, j)|^{p}\right)^{\frac{1}{p}}
$$

for any $x \in X$.

Moreover, define

$$
\Phi(x)=\frac{\|x\|^{p}}{p} \quad \text { and } \quad J(x)=\sum_{j=1}^{n} \sum_{i=1}^{m} F((i, j), x(i, j)), \quad \forall x \in X,
$$

where

$$
F((i, j), \xi)=\int_{0}^{\xi} f((i, j), \tau) d \tau, \quad \forall((i, j), \xi) \in \mathbb{Z}(1, m) \times \mathbb{Z}(1, n) \times \mathbb{R} .
$$

Obviously, $\Phi$ and $J$ are two functionals of class $C^{1}(X, \mathbb{R})$ and, for any $x, z \in X$,

$$
\begin{aligned}
\Phi^{\prime}(x)(z)= & \sum_{j=1}^{n} \sum_{i=1}^{m}\left\{-\Delta_{1}\left[\phi_{p}\left(\Delta_{1} x(i-1, j)\right)\right]-\Delta_{2}\left[\phi_{p}\left(\Delta_{2} x(i, j-1)\right)\right]\right. \\
& \left.+q(i, j) \phi_{p}(x(i, j))\right\} z(i, j), \\
J^{\prime}(x)(z)= & \sum_{j=1}^{n} \sum_{i=1}^{m} f((i, j), x(i, j)) z(i, j) .
\end{aligned}
$$


Therefore, for any $x, z \in X$,

$$
\begin{aligned}
(\Phi-\lambda J)^{\prime}(x)(z)= & \sum_{j=1}^{n} \sum_{i=1}^{m}\left\{-\Delta_{1}\left[\phi_{p}\left(\Delta_{1} x(i-1, j)\right)\right]-\Delta_{2}\left[\phi_{p}\left(\Delta_{2} x(i, j-1)\right)\right]\right. \\
& \left.+q(i, j) \phi_{p}(x(i, j))-\lambda f((i, j), x(i, j))\right\} z(i, j) .
\end{aligned}
$$

Remark 2.1 Clearly, $x$ is a critical point of the functional $\Phi-\lambda J$ in $X$ if and only if it is a solution of the problem $\left(S_{\lambda}^{f, q}\right)$. Then we transform the problem of seeking the solutions of $\left(S_{\lambda}^{f, q}\right)$ into looking for the critical points of $\Phi-\lambda J$ in $X$.

Put

$$
q_{*}=\min _{\substack{i \in \mathbb{Z}(1, m) \\ j \in \mathbb{Z}(1, n)}}\{q(i, j)\}, \quad q^{*}=\max _{\substack{i \in \mathbb{Z}(1, m) \\ j \in \mathbb{Z}(1, n)}}\{q(i, j)\} .
$$

According to Proposition 1 of [39], for any $x \in X$, we have

$$
\begin{aligned}
& \max _{\substack{i \in \mathbb{Z}(1, m) \\
j \in \mathbb{Z}(1, n)}}\{|x(i, j)|\} \\
& \quad \leq \frac{(m+n+2)^{\frac{p-1}{p}}}{4}\left(\sum_{j=1}^{n} \sum_{i=1}^{m+1}\left|\Delta_{1} x(i-1, j)\right|^{p}+\sum_{i=1}^{m} \sum_{j=1}^{n+1}\left|\Delta_{2} x(i, j-1)\right|^{p}\right)^{\frac{1}{p}} .
\end{aligned}
$$

Then we obtain the following result.

Lemma 2.1 For all $x \in X$, the inequality

$$
\max _{\substack{i \in \mathbb{Z}(1, m) \\ j \in \mathbb{Z}(1, n)}}\{|x(i, j)|\} \leq \frac{(m+n+2)^{\frac{p-1}{p}}}{\left[4^{p}+q_{*}(m+n+2)^{p-1}\right]^{\frac{1}{p}}}\|x\|
$$

holds.

Proof Owing to (2.2), we infer

$$
\begin{aligned}
\|x\|^{p} & =\sum_{j=1}^{n} \sum_{i=1}^{m+1}\left|\Delta_{1} x(i-1, j)\right|^{p}+\sum_{i=1}^{m} \sum_{j=1}^{n+1}\left|\Delta_{2} x(i, j-1)\right|^{p}+\sum_{j=1}^{n} \sum_{i=1}^{m} q(i, j)|x(i, j)|^{p} \\
& \geq \frac{4^{p}}{(m+n+2)^{p-1}}\left(\max _{\substack{i \in \mathbb{Z}(1, m) \\
j \in \mathbb{Z}(1, n)}}\{|x(i, j)|\}\right)^{p}+q_{*} \sum_{j=1}^{n} \sum_{i=1}^{m}|x(i, j)|^{p} \\
& \geq \frac{4^{p}}{(m+n+2)^{p-1}}\left(\max _{\substack{i \in \mathbb{Z}(1, m) \\
j \in \mathbb{Z}(1, n)}}\{|x(i, j)|\}\right)^{p}+q_{*}\left(\max _{\substack{i \in \mathbb{Z}(1, m) \\
j \in \mathbb{Z}(1, n)}}\{|x(i, j)|\}\right)^{p} \\
& =\frac{4^{p}+q_{*}(m+n+2)^{p-1}}{(m+n+2)^{p-1}}\left(\max _{\substack{i \in \mathbb{Z}(1, m) \\
j \in \mathbb{Z}(1, n)}}\{|x(i, j)|\}\right)^{p} .
\end{aligned}
$$


Therefore,

$$
\max _{\substack{i \in \mathbb{Z}(1, m) \\ j \in \mathbb{Z}(1, n)}}\{|x(i, j)|\} \leq \frac{(m+n+2)^{\frac{p-1}{p}}}{\left[4^{p}+q_{*}(m+n+2)^{p-1}\right]^{\frac{1}{p}}}\|x\| .
$$

For later convenience, we define another norm:

$$
\|x\|_{p}=\left(\sum_{j=1}^{n} \sum_{i=1}^{m}|x(i, j)|^{p}\right)^{\frac{1}{p}}, \quad \forall x \in X
$$

Since $X$ is an $m n$-dimensional space, the norms $\|\cdot\|$ and $\|\cdot\|_{p}$ are equivalent. To be specific, we have the following numerical estimation.

Lemma 2.2 For all $x \in X$, one has

$$
\frac{\left[4^{p}+q_{*} m n(m+n+2)^{p-1}\right]^{\frac{1}{p}}}{(m n)^{\frac{1}{p}}(m+n+2)^{\frac{p-1}{p}}}\|x\|_{p} \leq\|x\| \leq\left(2^{p+1}+q^{*}\right)^{\frac{1}{p}}\|x\|_{p} .
$$

Proof On the one hand, from (2.2) we have

$$
\begin{aligned}
\|x\|^{p} & =\sum_{j=1}^{n} \sum_{i=1}^{m+1}\left|\Delta_{1} x(i-1, j)\right|^{p}+\sum_{i=1}^{m} \sum_{j=1}^{n+1}\left|\Delta_{2} x(i, j-1)\right|^{p}+\sum_{j=1}^{n} \sum_{i=1}^{m} q(i, j)|x(i, j)|^{p} \\
& \geq \frac{4^{p}}{(m+n+2)^{p-1}}\left(\max _{\substack{i \in \mathbb{Z}(1, m) \\
j \in \mathbb{Z}(1, n)}}\{|x(i, j)|\}\right)^{p}+q_{*}\|x\|_{p}^{p} \\
& \geq \frac{4^{p}}{(m+n+2)^{p-1}}|x(i, j)|^{p}+q_{*}\|x\|_{p}^{p}
\end{aligned}
$$

for any $(i, j) \in \mathbb{Z}(1, m) \times \mathbb{Z}(1, n)$. This implies that

$$
|x(i, j)|^{p} \leq \frac{(m+n+2)^{p-1}}{4^{p}}\left[\|x\|^{p}-q_{*}\|x\|_{p}^{p}\right], \quad \forall(i, j) \in \mathbb{Z}(1, m) \times \mathbb{Z}(1, n) .
$$

Hence,

$$
\begin{aligned}
\|x\|_{p}^{p} & =\sum_{j=1}^{n} \sum_{i=1}^{m}|x(i, j)|^{p} \\
& \leq \sum_{j=1}^{n} \sum_{i=1}^{m} \frac{(m+n+2)^{p-1}}{4^{p}}\left[\|x\|^{p}-q_{*}\|x\|_{p}^{p}\right] \\
& =\frac{m n(m+n+2)^{p-1}}{4^{p}}\left[\|x\|^{p}-q_{*}\|x\|_{p}^{p}\right] \\
& =\frac{m n(m+n+2)^{p-1}}{4^{p}}\|x\|^{p}-\frac{q_{*} m n(m+n+2)^{p-1}}{4^{p}}\|x\|_{p}^{p},
\end{aligned}
$$

that is,

$$
\left[1+\frac{q_{*} m n(m+n+2)^{p-1}}{4^{p}}\right]\|x\|_{p}^{p} \leq \frac{m n(m+n+2)^{p-1}}{4^{p}}\|x\|^{p} .
$$


Therefore,

$$
\frac{\left[4^{p}+q_{*} m n(m+n+2)^{p-1}\right]^{\frac{1}{p}}}{(m n)^{\frac{1}{p}}(m+n+2)^{\frac{p-1}{p}}}\|x\|_{p} \leq\|x\| .
$$

On the other hand, for every $(i, j) \in \mathbb{Z}(1, m+1) \times \mathbb{Z}(1, n)$, we infer

$$
\left|\Delta_{1} x(i-1, j)\right|^{p} \leq(|x(i, j)|+|x(i-1, j)|)^{p} \leq 2^{p-1}\left(|x(i, j)|^{p}+|x(i-1, j)|^{p}\right),
$$

where the last inequality is due to the convexity property of the function $\phi(t)=t^{p}(t \geq 0)$. Thus,

$$
\begin{aligned}
\sum_{j=1}^{n} \sum_{i=1}^{m+1}\left|\Delta_{1} x(i-1, j)\right|^{p} & \leq 2^{p-1} \sum_{j=1}^{n} \sum_{i=1}^{m+1}\left(|x(i, j)|^{p}+|x(i-1, j)|^{p}\right) \\
& =2^{p-1}\left(\sum_{j=1}^{n} \sum_{i=1}^{m+1}|x(i, j)|^{p}+\sum_{j=1}^{n} \sum_{i=1}^{m+1}|x(i-1, j)|^{p}\right) \\
& =2^{p-1}\left(\sum_{j=1}^{n} \sum_{i=1}^{m}|x(i, j)|^{p}+\sum_{j=1}^{n} \sum_{i=1}^{m}|x(i, j)|^{p}\right) \\
& =2^{p} \sum_{j=1}^{n} \sum_{i=1}^{m}|x(i, j)|^{p} \\
& =2^{p}\|x\|_{p}^{p} .
\end{aligned}
$$

In the same way we get

$$
\sum_{i=1}^{m} \sum_{j=1}^{n+1}\left|\Delta_{2} x(i, j-1)\right|^{p} \leq 2^{p}\|x\|_{p}^{p}
$$

Besides,

$$
\sum_{j=1}^{n} \sum_{i=1}^{m} q(i, j)|x(i, j)|^{p} \leq q^{*} \sum_{j=1}^{n} \sum_{i=1}^{m}|x(i, j)|^{p}=q^{*}\|x\|_{p}^{p}
$$

Summarizing,

$$
\|x\|^{p} \leq 2^{p}\|x\|_{p}^{p}+2^{p}\|x\|_{p}^{p}+q^{*}\|x\|_{p}^{p}=\left(2^{p+1}+q^{*}\right)\|x\|_{p}^{p},
$$

that is,

$$
\|x\| \leq\left(2^{p+1}+q^{*}\right)^{\frac{1}{p}}\|x\|_{p}
$$

which yields our conclusion.

In order to obtain positive solutions of problem $\left(S_{\lambda}^{f, q}\right)$, we establish the following strong maximum principle. 
Lemma 2.3 Fix $x \in X$ such that, for any $(i, j) \in \mathbb{Z}(1, m) \times \mathbb{Z}(1, n)$, either

$$
\begin{aligned}
& x(i, j)>0 \text { or } \\
& -\Delta_{1}\left[\phi_{p}\left(\Delta_{1} x(i-1, j)\right)\right]-\Delta_{2}\left[\phi_{p}\left(\Delta_{2} x(i, j-1)\right)\right]+q(i, j) \phi_{p}(x(i, j)) \geq 0 .
\end{aligned}
$$

Then either $x(i, j)>0$ for all $(i, j) \in \mathbb{Z}(1, m) \times \mathbb{Z}(1, n)$ or $x \equiv 0$.

Proof Fix $x \in X$ satisfying (2.5). Let $\theta \in \mathbb{Z}(1, m), \omega \in \mathbb{Z}(1, n)$ such that

$$
x(\theta, \omega)=\min \{x(i, j): i \in \mathbb{Z}(1, m), j \in \mathbb{Z}(1, n)\} .
$$

If $x(\theta, \omega)>0$, then $x(i, j)>0$ for all $i \in \mathbb{Z}(1, m), j \in \mathbb{Z}(1, n)$, and the proof is finished.

If $x(\theta, \omega) \leq 0$, then $x(\theta, \omega)=\min \{x(i, j): i \in \mathbb{Z}(0, m+1), j \in \mathbb{Z}(0, n+1)\}$. At this point, it is easy to see that $\Delta_{1} x(\theta-1, \omega)=x(\theta, \omega)-x(\theta-1, \omega) \leq 0$ and $\Delta_{1} x(\theta, \omega)=x(\theta+1, \omega)-$ $x(\theta, \omega) \geq 0$. Since $\phi_{p}(s)$ is increasing in $s$, and $\phi_{p}(0)=0$, one has

$$
\phi_{p}\left(\Delta_{1} x(\theta-1, \omega)\right) \leq 0 \leq \phi_{p}\left(\Delta_{1} x(\theta, \omega)\right)
$$

which implies that

$$
\Delta_{1}\left[\phi_{p}\left(\Delta_{1} x(\theta-1, \omega)\right)\right] \geq 0
$$

Similarly,

$$
\Delta_{2}\left[\phi_{p}\left(\Delta_{2} x(\theta, \omega-1)\right)\right] \geq 0
$$

Thus,

$$
\Delta_{1}\left[\phi_{p}\left(\Delta_{1} x(\theta-1, \omega)\right)\right]+\Delta_{2}\left[\phi_{p}\left(\Delta_{2} x(\theta, \omega-1)\right)\right] \geq 0 .
$$

On the other hand, in view of (2.5), we infer

$$
\Delta_{1}\left[\phi_{p}\left(\Delta_{1} x(\theta-1, \omega)\right)\right]+\Delta_{2}\left[\phi_{p}\left(\Delta_{2} x(\theta, \omega-1)\right)\right] \leq q(\theta, \omega) \phi_{p}(x(\theta, \omega)) \leq 0 .
$$

Combining (2.6) and (2.7), we have

$$
\Delta_{1}\left[\phi_{p}\left(\Delta_{1} x(\theta-1, \omega)\right)\right]+\Delta_{2}\left[\phi_{p}\left(\Delta_{2} x(\theta, \omega-1)\right)\right]=0,
$$

which yields

$$
\Delta_{1}\left[\phi_{p}\left(\Delta_{1} x(\theta-1, \omega)\right)\right]=\Delta_{2}\left[\phi_{p}\left(\Delta_{2} x(\theta, \omega-1)\right)\right]=0,
$$

namely,

$$
\left\{\begin{array}{l}
\phi_{p}\left(\Delta_{1} x(\theta, \omega)\right)=\phi_{p}\left(\Delta_{1} x(\theta-1, \omega)\right)=0, \\
\phi_{p}\left(\Delta_{2} x(\theta, \omega)\right)=\phi_{p}\left(\Delta_{2} x(\theta, \omega-1)\right)=0 .
\end{array}\right.
$$


Therefore,

$$
\left\{\begin{array}{l}
x(\theta+1, \omega)=x(\theta, \omega)=x(\theta-1, \omega), \\
x(\theta, \omega+1)=x(\theta, \omega)=x(\theta, \omega-1) .
\end{array}\right.
$$

If $\theta+1=m+1$, we get $x(\theta, \omega)=0$. Otherwise, $\theta+1 \in \mathbb{Z}(1, m)$. Replacing $\theta$ by $\theta+1$, we have $x(\theta+2, \omega)=x(\theta+1, \omega)$. Continuing this process $m+1-\theta$ times, we obtain $x(\theta, \omega)=x(\theta+1, \omega)=x(\theta+2, \omega)=\cdots=x(m, \omega)=x(m+1, \omega)=0$. Analogously, we have $x(\theta, \omega)=x(\theta-1, \omega)=x(\theta-2, \omega)=\cdots=x(1, \omega)=x(0, \omega)=0$. Hence, $x(i, \omega)=0$ for each $i \in \mathbb{Z}(1, m)$. In the same way we can prove that $x \equiv 0$ and the conclusion of Lemma 2.3 holds.

\section{Main results}

Denote

$$
Q=\sum_{j=1}^{n} \sum_{i=1}^{m} q(i, j)
$$

Our main result is the following.

Theorem 3.1 Assume that there exist four positive constants $c, d, \mu, \alpha$ satisfying $\alpha<p$ and $d^{p}>\frac{\left[4^{p}+q_{*}(m+n+2)^{p-1}\right] c^{p}}{(2 m+2 n+Q)(m+n+2)^{p-1}}$ such that

$\left(A_{1}\right) \max _{((i, j)), \xi) \in \mathbb{Z}(1, m) \times \mathbb{Z}(1, n) \times[-c, c]} F((i, j), \xi)<\frac{\left.\left[4^{p}+q_{*}(m+n+2)\right)^{p-1}\right] c^{p} \sum_{j=1}^{n} \sum_{i=1}^{m} F((i, j), d)}{m n\left\{\left[4^{p}+q_{*}(m+n+2)^{p-1}\right] c^{p}+(2 m+2 n+Q)(m+n+2)^{p-1} d^{p}\right\}} ;$

$\left(A_{2}\right) F((i, j), \xi) \leq \mu\left(1+|\xi|^{\alpha}\right), \forall((i, j), \xi) \in \mathbb{Z}(1, m) \times \mathbb{Z}(1, n) \times \mathbb{R}$.

Furthermore, put

$$
\begin{aligned}
& \lambda_{1}=\frac{p m n(m+n+2)^{p-1} \max _{((i, j), \xi) \in \mathbb{Z}(1, m) \times \mathbb{Z}(1, n) \times[-c, c]} F((i, j), \xi)}{\left[4^{p}+q_{*}(m+n+2)^{p-1}\right] c^{p}}, \\
& \lambda_{2}=\frac{p\left[\sum_{j=1}^{n} \sum_{i=1}^{m} F((i, j), d)-m n \max _{((i, j), \xi) \in \mathbb{Z}(1, m) \times \mathbb{Z}(1, n) \times[-c, c]} F((i, j), \xi)\right]}{(2 m+2 n+Q) d^{p}} .
\end{aligned}
$$

Then, for each $\lambda \in \Lambda_{1}=\left(\frac{1}{\lambda_{2}}, \frac{1}{\lambda_{1}}\right)$, problem $\left(S_{\lambda}^{f, q}\right)$ possesses at least three solutions in $X$.

Moreover, put

$$
\begin{aligned}
a= & (2 m+2 n+Q)\left[4^{p}+q_{*}(m+n+2)^{p-1}\right](c d)^{p}, \\
b= & p\left[4^{p}+q_{*}(m+n+2)^{p-1}\right] c^{p} \sum_{j=1}^{n} \sum_{i=1}^{m} F((i, j), d) \\
& -p m n(2 m+2 n+Q)(m+n+2)^{p-1} d^{p} \max _{((i, j)), \xi) \in \mathbb{Z}(1, m) \times \mathbb{Z}(1, n) \times[-c, c]} F((i, j), \xi) .
\end{aligned}
$$

Then, for any $h>1$, there exist an open interval $\Lambda_{2} \subseteq\left[0, \frac{a}{b} h\right]$ and a real number $\sigma>0$ such that, for each $\lambda \in \Lambda_{2}$, problem $\left(S_{\lambda}^{f, q}\right)$ possesses at least three solutions in $X$ and their norms are all less than $\sigma$. 
Remark 3.1 From $\left(A_{1}\right)$ it follows that

$$
\begin{aligned}
m n(2 m+2 n+Q)(m+n+2)^{p-1} d^{p} \max _{((i, j), \xi) \in \mathbb{Z}(1, m) \times \mathbb{Z}(1, n) \times[-c, c]} F((i, j), \xi) \\
<\left[4^{p}+q_{*}(m+n+2)^{p-1}\right] \\
\quad \times c^{p}\left[\sum_{j=1}^{n} \sum_{i=1}^{m} F((i, j), d)-m n \max _{((i, j), \xi) \in \mathbb{Z}(1, m) \times \mathbb{Z}(1, n) \times[-c, c]} F((i, j), \xi)\right] .
\end{aligned}
$$

Then

$$
\begin{aligned}
& \frac{p m n(m+n+2)^{p-1} \max _{((i, j), \xi) \in \mathbb{Z}(1, m) \times \mathbb{Z}(1, n) \times[-c, c]} F((i, j), \xi)}{\left[4^{p}+q_{*}(m+n+2)^{p-1}\right] c^{p}} \\
& <\frac{p\left[\sum_{j=1}^{n} \sum_{i=1}^{m} F((i, j), d)-m n \max _{((i, j), \xi) \in \mathbb{Z}(1, m) \times \mathbb{Z}(1, n) \times[-c, c]} F((i, j), \xi)\right]}{(2 m+2 n+Q) d^{p}} .
\end{aligned}
$$

That is, $\lambda_{1}<\lambda_{2}$, which indicates that the interval $\left(\frac{1}{\lambda_{2}}, \frac{1}{\lambda_{1}}\right)$ is well-defined.

Remark 3.2 In view of assumption $\left(A_{1}\right)$, we infer

$$
\begin{aligned}
& {\left[4^{p}+q_{*}(m+n+2)^{p-1}\right] c^{p} \sum_{j=1}^{n} \sum_{i=1}^{m} F((i, j), d)} \\
& >m n(2 m+2 n+Q)(m+n+2)^{p-1} d^{p} \max _{((i, j), \xi) \in \mathbb{Z}(1, m) \times \mathbb{Z}(1, n) \times[-c, c]} F((i, j), \xi),
\end{aligned}
$$

so $b>0$ and $\left[0, \frac{a}{b} h\right]$ is a well-defined interval.

Remark 3.3 When $f: \mathbb{Z}(1, m) \times \mathbb{Z}(1, n) \times \mathbb{R} \rightarrow \mathbb{R}$ is a nonnegative function, Lemma 2.3 guarantees that every solution mentioned in Theorem 3.1 is either positive or zero.

Proof of Theorem 3.1 Since $X$ is a finite-dimensional real Banach space, $X$ is separable and reflexive. From the definitions in (2.1) of $\Phi$ and $J$, we know that $\Phi: X \rightarrow \mathbb{R}$ is a nonnegative continuously Gâteaux differentiable and sequentially weakly lower semicontinuous functional whose Gâteaux derivative admits a continuous inverse on $X^{*}$, and $J: X \rightarrow \mathbb{R}$ is a continuously Gâteaux differentiable functional whose Gâteaux derivative is compact. Choose $x_{0}(i, j)=0$ for each $(i, j) \in \mathbb{Z}(0, m+1) \times \mathbb{Z}(0, n+1)$, it is clear that $x_{0} \in X$ and $\Phi\left(x_{0}\right)=0=J\left(x_{0}\right)$.

According to the assumption $\left(A_{2}\right)$ and Lemma 2.2, we deduce

$$
\begin{aligned}
\Phi(x)-\lambda J(x) & =\frac{1}{p}\|x\|^{p}-\lambda \sum_{j=1}^{n} \sum_{i=1}^{m} F((i, j), x(i, j)) \\
& \geq \frac{4^{p}+q_{*} m n(m+n+2)^{p-1}}{p m n(m+n+2)^{p-1}}\|x\|_{p}^{p}-\lambda \sum_{j=1}^{n} \sum_{i=1}^{m} \mu\left(1+|x(i, j)|^{\alpha}\right)
\end{aligned}
$$




$$
\begin{aligned}
& =\frac{4^{p}+q_{*} m n(m+n+2)^{p-1}}{p m n(m+n+2)^{p-1}} \sum_{j=1}^{n} \sum_{i=1}^{m}|x(i, j)|^{p}-\lambda \mu \sum_{j=1}^{n} \sum_{i=1}^{m}\left(1+|x(i, j)|^{\alpha}\right) \\
& =\sum_{j=1}^{n} \sum_{i=1}^{m}\left[\frac{4^{p}+q_{*} m n(m+n+2)^{p-1}}{p m n(m+n+2)^{p-1}}|x(i, j)|^{p}-\lambda \mu|x(i, j)|^{\alpha}-\lambda \mu\right]
\end{aligned}
$$

for any $x \in X$ and $\lambda \geq 0$. Bearing in mind $\alpha<p$, one has

$$
\lim _{\|x\| \rightarrow+\infty}[\Phi(x)-\lambda J(x)]=+\infty, \quad \forall \lambda \in[0,+\infty)
$$

namely, the condition (i) of Lemma 1.1 is fulfilled.

For the condition (ii), we put

$$
\begin{aligned}
& r=\frac{\left[4^{p}+q_{*}(m+n+2)^{p-1}\right] c^{p}}{p(m+n+2)^{p-1}}, \\
& x_{1}(i, j)= \begin{cases}0, & \text { if } i=0, j \in \mathbb{Z}(0, n+1) \text { or } i=m+1, j \in \mathbb{Z}(0, n+1), \\
d, & \text { if }(i, j) \in \mathbb{Z}(1, m) \times \mathbb{Z}(1, n), \\
0, & \text { if } j=0, i \in \mathbb{Z}(0, m+1) \text { or } j=n+1, i \in \mathbb{Z}(0, m+1) .\end{cases}
\end{aligned}
$$

It follows that $x_{1} \in X$ and

$$
\begin{aligned}
& \Phi\left(x_{1}\right)=\frac{\left\|x_{1}\right\|^{p}}{p}=\frac{2 m+2 n+Q}{p} d^{p}, \\
& J\left(x_{1}\right)=\sum_{j=1}^{n} \sum_{i=1}^{m} F\left((i, j), x_{1}(i, j)\right)=\sum_{j=1}^{n} \sum_{i=1}^{m} F((i, j), d) .
\end{aligned}
$$

In view of $d^{p}>\frac{\left[4^{p}+q_{*}(m+n+2)^{p-1}\right] c^{p}}{(2 m+2 n+Q)(m+n+2)^{p-1}}$, we have

$$
\Phi\left(x_{1}\right)=\frac{2 m+2 n+Q}{p} d^{p}>\frac{\left[4^{p}+q_{*}(m+n+2)^{p-1}\right] c^{p}}{p(m+n+2)^{p-1}}=r,
$$

which means that the condition (ii) of Lemma 1.1 is satisfied.

Next, we verify the condition (iii) of Lemma 1.1. By direct computation, we get

$$
\frac{r}{r+\Phi\left(x_{1}\right)} J\left(x_{1}\right)=\frac{\left[4^{p}+q_{*}(m+n+2)^{p-1}\right] c^{p} \sum_{j=1}^{n} \sum_{i=1}^{m} F((i, j), d)}{\left[4^{p}+q_{*}(m+n+2)^{p-1}\right] c^{p}+(2 m+2 n+Q)(m+n+2)^{p-1} d^{p}} .
$$

On the other hand, for any $x \in \Phi^{-1}(-\infty, r]$, i.e., $\Phi(x) \leq r$, we infer

$$
\begin{aligned}
|x(i, j)| & \leq \max _{\substack{i \in \mathbb{Z}(1, m) \\
j \in \mathbb{Z}(1, n)}}\{|x(i, j)|\} \\
& \leq \frac{(m+n+2)^{\frac{p-1}{p}}}{\left[4^{p}+q_{*}(m+n+2)^{p-1}\right]^{\frac{1}{p}}}\|x\| \leq \frac{(m+n+2)^{\frac{p-1}{p}}(p r)^{\frac{1}{p}}}{\left[4^{p}+q_{*}(m+n+2)^{p-1}\right]^{\frac{1}{p}}}=c
\end{aligned}
$$

for every $(i, j) \in \mathbb{Z}(1, m) \times \mathbb{Z}(1, n)$. This leads to

$$
\Phi^{-1}(-\infty, r] \subseteq\{x \in X:|x(i, j)| \leq c, \forall(i, j) \in \mathbb{Z}(1, m) \times \mathbb{Z}(1, n)\} .
$$


Hence, this along with assumption $\left(A_{1}\right)$ yields

$$
\begin{aligned}
\sup _{x \in \Phi^{-1}(-\infty, r)} J(x) & \leq \sup _{x \in\{x \in X:|x(i, j)| \leq c, \forall(i, j) \in \mathbb{Z}(1, m) \times \mathbb{Z}(1, n)\}} \sum_{j=1}^{n} \sum_{i=1}^{m} F((i, j), x(i, j)) \\
& \leq m n \max _{((i, j), \xi) \in \mathbb{Z}(1, m) \times \mathbb{Z}(1, n) \times[-c, c]} F((i, j), \xi) \\
& <\frac{\left[4^{p}+q_{*}(m+n+2)^{p-1}\right] c^{p} \sum_{j=1}^{n} \sum_{i=1}^{m} F((i, j), d)}{\left[4^{p}+q_{*}(m+n+2)^{p-1}\right] c^{p}+(2 m+2 n+Q)(m+n+2)^{p-1} d^{p}} \\
& =\frac{r}{r+\Phi\left(x_{1}\right)} J\left(x_{1}\right)
\end{aligned}
$$

for any $x \in X$. The condition (iii) of Lemma 1.1 is verified.

Note that

$$
\begin{aligned}
& \frac{\Phi\left(x_{1}\right)}{J\left(x_{1}\right)-\sup _{x \in \Phi^{-1}(-\infty, r)}{ }^{w} J(x)} \\
& \leq \frac{(2 m+2 n+Q) d^{p}}{p\left[\sum_{j=1}^{n} \sum_{i=1}^{m} F((i, j), d)-m n \max _{((i, j), \xi) \in \mathbb{Z}(1, m) \times \mathbb{Z}(1, n) \times[-c, c]} F((i, j), \xi)\right]}=\frac{1}{\lambda_{2}}, \\
& \frac{r}{\sup _{x \in \bar{\Phi}^{-1}(-\infty, r)} w(x)} \geq \frac{\left[4^{p}+q_{*}(m+n+2)^{p-1}\right] c^{p}}{p m n(m+n+2)^{p-1} \max _{((i, j), \xi) \in \mathbb{Z}(1, m) \times \mathbb{Z}(1, n) \times[-c, c]} F((i, j), \xi)}=\frac{1}{\lambda_{1}} .
\end{aligned}
$$

According to Lemma 1.1 and Remark 2.1, for any $\lambda \in \Lambda_{1}=\left(\frac{1}{\lambda_{2}}, \frac{1}{\lambda_{1}}\right)$, problem $\left(S_{\lambda}^{f, q}\right)$ possesses at least three solutions in $X$.

Moreover, for any $h>1$, it follows from the expressions of $a$ and $b$ that

$$
\frac{h r}{\frac{r J\left(x_{1}\right)}{\Phi\left(x_{1}\right)}-\sup _{x \in \bar{\Phi}^{-1}(-\infty, r)}{ }^{w} J(x)} \leq \frac{a}{b} h
$$

By Lemma 1.1 and Remark 2.1, for any $h>1$, there exist an open interval $\Lambda_{2} \subseteq\left[0, \frac{a}{b} h\right]$ and a real number $\sigma>0$ such that, for each $\lambda \in \Lambda_{2},\left(S_{\lambda}^{f, q}\right)$ possesses at least three solutions in $X$ and their norms all are less than $\sigma$. This completes the proof of Theorem 3.1.

The following result, as a direct consequence of Theorem 3.1, ensures the existence of at least two positive solutions for problem $\left(S_{\lambda}^{f, q}\right)$.

Corollary 3.2 Iff $((i, j), 0) \geq 0$ for all $(i, j) \in \mathbb{Z}(1, m) \times \mathbb{Z}(1, n)$, and there exist four positive constants $c, d, \mu, \alpha$ with $\alpha<p$ and $d^{p}>\frac{\left[4^{p}+q_{*}(m+n+2)^{p-1}\right] c^{p}}{(2 m+2 n+Q)(m+n+2)^{p-1}}$ such that

$\left(A_{1}^{*}\right)$

$$
\begin{aligned}
& \max _{((i, j), \xi) \in \mathbb{Z}(1, m) \times \mathbb{Z}(1, n) \times[0, c]} \int_{0}^{\xi} f((i, j), \tau) d \tau \\
& <\frac{\left[4^{p}+q_{*}(m+n+2)^{p-1}\right] c^{p} \sum_{j=1}^{n} \sum_{i=1}^{m} \int_{0}^{d} f((i, j), \tau) d \tau}{m n\left\{\left[4^{p}+q_{*}(m+n+2)^{p-1}\right] c^{p}+(2 m+2 n+Q)(m+n+2)^{p-1} d^{p}\right\}}
\end{aligned}
$$

$\left(A_{2}^{*}\right) \int_{0}^{\xi} f((i, j), \tau) d \tau \leq \mu\left(1+|\xi|^{\alpha}\right), \forall((i, j), \xi) \in \mathbb{Z}(1, m) \times \mathbb{Z}(1, n) \times(0,+\infty)$. 
Furthermore, denote

$$
\begin{aligned}
& \lambda_{1}=\frac{p m n(m+n+2)^{p-1} \max _{((i, j)), \xi) \in \mathbb{Z}(1, m) \times \mathbb{Z}(1, n) \times[0, c]} \int_{0}^{\xi} f((i, j), \tau) d \tau}{\left[4^{p}+q_{*}(m+n+2)^{p-1}\right] c^{p}}, \\
& \lambda_{2}=\frac{p\left[\sum_{j=1}^{n} \sum_{i=1}^{m} \int_{0}^{d} f((i, j), \tau) d \tau-m n \max _{\left.((i, j)), \xi) \in \mathbb{Z}(1, m) \times \mathbb{Z}(1, n) \times[0, c] \int_{0}^{\xi} f((i, j), \tau) d \tau\right]} .\right.}{(2 m+2 n+Q) d^{p}} .
\end{aligned}
$$

Then, for any $\lambda \in \Lambda_{1}=\left(\frac{1}{\lambda_{2}}, \frac{1}{\lambda_{1}}\right)$, problem $\left(S_{\lambda}^{f, q}\right)$ has at least two positive solutions in $X$.

Moreover, denote

$$
\begin{aligned}
a= & (2 m+2 n+Q)\left[4^{p}+q_{*}(m+n+2)^{p-1}\right](c d)^{p}, \\
b= & p\left[4^{p}+q_{*}(m+n+2)^{p-1}\right] c^{p} \sum_{j=1}^{n} \sum_{i=1}^{m} \int_{0}^{d} f((i, j), \tau) d \tau \\
& -p m n(2 m+2 n+Q)(m+n+2)^{p-1} d^{p} \max _{((i, j), \xi) \in \mathbb{Z}(1, m) \times \mathbb{Z}(1, n) \times[0, c]} \int_{0}^{\xi} f((i, j), \tau) d \tau .
\end{aligned}
$$

Then, for any $h>1$, there exist an open interval $\Lambda_{2} \subseteq\left[0, \frac{a}{b} h\right]$ and a positive real number $\sigma$ such that, for each $\lambda \in \Lambda_{2}$, problem $\left(S_{\lambda}^{f, q}\right)$ has at least two positive solutions in $X$ and their norms are all less than $\sigma$.

Proof For any $(i, j) \in \mathbb{Z}(1, m) \times \mathbb{Z}(1, n)$ and $t \in \mathbb{R}$, we put

$$
\begin{aligned}
& f^{*}((i, j), t)= \begin{cases}f((i, j), t), & t>0, \\
f((i, j), 0), & t \leq 0,\end{cases} \\
& F^{*}((i, j), t)=\int_{0}^{t} f^{*}((i, j), \tau) d \tau .
\end{aligned}
$$

Therefore,

$$
\begin{aligned}
& \max _{((i, j), \xi) \in \mathbb{Z}(1, m) \times \mathbb{Z}(1, n) \times[-c, c]} F^{*}((i, j), \xi)=\max _{((i, j), \xi) \in \mathbb{Z}(1, m) \times \mathbb{Z}(1, n) \times[0, c]} \int_{0}^{\xi} f((i, j), \tau) d \tau, \\
& \sum_{j=1}^{n} \sum_{i=1}^{m} F^{*}((i, j), d)=\sum_{j=1}^{n} \sum_{i=1}^{m} \int_{0}^{d} f((i, j), \tau) d \tau .
\end{aligned}
$$

In view of hypotheses $\left(A_{1}^{*}\right)$ and $\left(A_{2}^{*}\right)$, the conclusion of Theorem 3.1 holds for problem $\left(S_{\lambda}^{f^{*}, q}\right)$. Further, by applying Lemma 2.3 , we find that problem $\left(S_{\lambda}^{f^{*}, q}\right)$ admits at least two positive solutions when $\lambda$ belongs to intervals $\Lambda_{1}$ and $\Lambda_{2}$, respectively, which are exactly positive solutions of problem $\left(S_{\lambda}^{f, q}\right)$. The proof of Corollary 3.2 is complete.

Next, we study a special case in which $f$ has separated variables. Specifically, we consider the following problem, namely $\left(S_{\lambda}^{\omega g, q}\right)$ :

$$
\begin{aligned}
& -\Delta_{1}\left[\phi_{p}\left(\Delta_{1} x(i-1, j)\right)\right]-\Delta_{2}\left[\phi_{p}\left(\Delta_{2} x(i, j-1)\right)\right]+q(i, j) \phi_{p}(x(i, j))=\lambda \omega(i, j) g(x(i, j)), \\
& (i, j) \in \mathbb{Z}(1, m) \times \mathbb{Z}(1, n),
\end{aligned}
$$


with Dirichlet boundary conditions

$$
\begin{aligned}
& x(i, 0)=x(i, n+1)=0, \quad i \in \mathbb{Z}(0, m+1), \\
& x(0, j)=x(m+1, j)=0, \quad j \in \mathbb{Z}(0, n+1),
\end{aligned}
$$

where $\omega: \mathbb{Z}(1, m) \times \mathbb{Z}(1, n) \rightarrow \mathbb{R}$ is nonnegative and non-zero, and $g:[0,+\infty) \rightarrow \mathbb{R}$ is a nonnegative continuous function.

Define

$$
W=\sum_{j=1}^{n} \sum_{i=1}^{m} \omega(i, j), \quad G(\xi)=\int_{0}^{\xi} g(s) d s
$$

Then we have the following result.

Corollary 3.3 Assume that there exist four positive constants $c, d, \eta, \alpha$ satisfying $\alpha<p$ and $d^{p}>\frac{\left[4^{p}+q_{*}(m+n+2)^{p-1}\right] c^{p}}{(2 m+2 n+Q)(m+n+2)^{p-1}}$ such that

$\left(A_{1}^{\prime}\right) \max _{(i, j) \in \mathbb{Z}(1, m) \times \mathbb{Z}(1, n)} \omega(i, j)<\frac{\left[4^{p}+q_{*}(m+n+2)^{p-1}\right] c^{p} W G(d)}{m n\left\{\left[4^{p}+q_{*}(m+n+2)^{p-1}\right] c^{p}+(2 m+2 n+Q)(m+n+2)^{p-1} d^{p}\right\} G(c)} ;$

$\left(A_{2}^{\prime}\right) G(\xi) \leq \eta\left(1+|\xi|^{\alpha}\right), \forall \xi>0$.

Furthermore, denote

$$
\begin{aligned}
& \lambda_{1}=\frac{p m n(m+n+2)^{p-1} G(c) \max _{(i, j) \in \mathbb{Z}(1, m) \times \mathbb{Z}(1, n)} \omega(i, j)}{\left[4^{p}+q_{*}(m+n+2)^{p-1}\right] c^{p}}, \\
& \lambda_{2}=\frac{p\left[W G(d)-m n G(c) \max _{(i, j) \in \mathbb{Z}(1, m) \times \mathbb{Z}(1, n)} \omega(i, j)\right]}{(2 m+2 n+Q) d^{p}} .
\end{aligned}
$$

Then, for any $\lambda \in \Lambda_{1}=\left(\frac{1}{\lambda_{2}}, \frac{1}{\lambda_{1}}\right)$, problem $\left(S_{\lambda}^{\omega g g, q}\right)$ has at least two positive solutions in $X$.

Moreover, denote

$$
\begin{aligned}
a= & (2 m+2 n+Q)\left[4^{p}+q_{*}(m+n+2)^{p-1}\right](c d)^{p}, \\
b= & p\left[4^{p}+q_{*}(m+n+2)^{p-1}\right] c^{p} W G(d) \\
& -p m n(2 m+2 n+Q)(m+n+2)^{p-1} d^{p} G(c) \max _{(i, j) \in \mathbb{Z}(1, m) \times \mathbb{Z}(1, n)} \omega(i, j) .
\end{aligned}
$$

Then, for any $h>1$, there exist an open interval $\Lambda_{2} \subseteq\left[0, \frac{a}{b} h\right]$ and a positive real number $\sigma$ such that, for each $\lambda \in \Lambda_{2}$, problem $\left(S_{\lambda}^{\omega g, q}\right)$ has at least two positive solutions in $X$ and their norms are all less than $\sigma$.

Proof Set

$$
f((i, j), s)= \begin{cases}\omega(i, j) g(s), & s \geq 0, \\ \omega(i, j) g(0), & s<0,\end{cases}
$$

for any $(i, j) \in \mathbb{Z}(1, m) \times \mathbb{Z}(1, n)$ and $s \in \mathbb{R}$. It is easy to verify that

$$
f((i, j), 0)=\omega(i, j) g(0) \geq 0, \quad \forall(i, j) \in \mathbb{Z}(1, m) \times \mathbb{Z}(1, n),
$$




$$
\begin{aligned}
& \max _{((i, j), \xi) \in \mathbb{Z}(1, m) \times \mathbb{Z}(1, n) \times[0, c]} \int_{0}^{\xi} f((i, j), \tau) d \tau=G(c) \max _{(i, j) \in \mathbb{Z}(1, m) \times \mathbb{Z}(1, n)} \omega(i, j), \\
& \sum_{j=1}^{n} \sum_{i=1}^{m} \int_{0}^{d} f((i, j), \tau) d \tau=W G(d) .
\end{aligned}
$$

Besides, we take $\mu=\eta \max _{(i, j) \in \mathbb{Z}(1, m) \times \mathbb{Z}(1, n)} \omega(i, j)$. The conclusion follows from Corollary 3.2 and taking into account (3.1).

\section{An example}

To illustrate our results, we present a concrete example.

Example 4.1 Consider the problem $\left(S_{\lambda}^{\omega g, q}\right)$ and take $p=4, m=2, n=2, c=1, d=10, \eta=$ $e^{12}, \alpha=1$ and

$$
\begin{aligned}
& q(i, j)=i j, \quad \forall(i, j) \in \mathbb{Z}(1,2) \times \mathbb{Z}(1,2), \\
& \omega(i, j)=i+j, \quad \forall(i, j) \in \mathbb{Z}(1,2) \times \mathbb{Z}(1,2), \\
& g(s)= \begin{cases}s e^{s,} & 0 \leq s \leq 9, \\
9 e^{9}, & s>9 .\end{cases}
\end{aligned}
$$

Then we get $Q=9, W=12, q_{*}=1, \max _{(i, j) \in \mathbb{Z}(1,2) \times \mathbb{Z}(1,2)} \omega(i, j)=4$, and

$$
G(\xi)= \begin{cases}(\xi-1) e^{\xi}+1, & 0 \leq \xi \leq 9, \\ 9 e^{9} \xi-73 e^{9}+1, & \xi>9 .\end{cases}
$$

So $G(c)=1, G(d)=17 e^{9}+1$. Furthermore,

$$
\frac{\left[4^{p}+q_{*}(m+n+2)^{p-1}\right] c^{p}}{(2 m+2 n+Q)(m+n+2)^{p-1}}=\frac{472}{3672}<10^{4}=d^{p}
$$

and

$$
\frac{\left[4^{p}+q_{*}(m+n+2)^{p-1}\right] c^{p} W G(d)}{m n\left\{\left[4^{p}+q_{*}(m+n+2)^{p-1}\right] c^{p}+(2 m+2 n+Q)(m+n+2)^{p-1} d^{p}\right\} G(c)}=\frac{177\left(17 e^{9}+1\right)}{4,590,059} .
$$

Then the condition $\left(A_{1}^{\prime}\right)$ of Corollary 3.3 holds.

Due to (4.1), we have

$$
\begin{aligned}
& G(\xi)=(\xi-1) e^{\xi}+1 \leq 8 e^{9}+1<e^{12}(1+|\xi|)=\eta\left(1+|\xi|^{\alpha}\right), \quad \forall 0<\xi \leq 9 ; \\
& G(\xi)=9 e^{9} \xi-73 e^{9}+1<e^{12} \xi+e^{12}=e^{12}(1+|\xi|)=\eta\left(1+|\xi|^{\alpha}\right), \quad \forall \xi>9,
\end{aligned}
$$

which indicate

$$
G(\xi) \leq \eta\left(1+|\xi|^{\alpha}\right), \quad \forall \xi>0
$$

that is, the condition $\left(A_{2}^{\prime}\right)$ of Corollary 3.3 is fulfilled. 
Moreover,

$$
\begin{aligned}
& \lambda_{1}=\frac{p m n(m+n+2)^{p-1} G(c) \max _{(i, j) \in \mathbb{Z}(1, m) \times \mathbb{Z}(1, n)} \omega(i, j)}{\left[4^{p}+q_{*}(m+n+2)^{p-1}\right] c^{p}}=\frac{1728}{59}, \\
& \lambda_{2}=\frac{p\left[W G(d)-m n G(c) \max _{(i, j) \in \mathbb{Z}(1, m) \times \mathbb{Z}(1, n)} \omega(i, j)\right]}{(2 m+2 n+Q) d^{p}}=\frac{51 e^{9}-1}{10,625} .
\end{aligned}
$$

By Corollary 3.3, for any $\lambda \in \Lambda_{1}=\left(\frac{10,625}{51 e^{9}-1}, \frac{59}{1728}\right)$, the considered problem possesses at least two positive solutions in $X$.

Besides, $a$ and $b$ in Corollary 3.3 are

$$
a=80,240,000, \quad b=385,152 e^{9}-2,350,057,344,
$$

respectively. Therefore, for any $h>1$, there exist an open interval $\Lambda_{2} \subseteq\left[0, \frac{626,875}{3009 e^{9}-18,359,823} h\right]$ and a positive real number $\sigma$ such that, for each $\lambda \in \Lambda_{2}$, the considered problem has at least two positive solutions in $X$ and their norms are all less than $\sigma$.

In particular, we take $\lambda=0.03 \in \Lambda_{1}$. By a careful computation, we find that the considered problem admits at least two positive solutions $x_{1}=\left\{x_{1}(i, j)\right\}_{i \in \mathbb{Z}(0,3)} \in X$ and $x_{2}=$ $\left\{x_{2}(i, j)\right\}_{i \in \mathbb{Z}(0,3)} \in X$, where $j \in \mathbb{Z}(0,3)$

$$
\left(\begin{array}{l}
x_{1}(1,1) \\
x_{1}(1,2) \\
x_{1}(2,1) \\
x_{1}(2,2)
\end{array}\right)=\left(\begin{array}{c}
1 \\
1 \\
1 \\
\sqrt[3]{\frac{27 e^{9}+6 e-275}{150}}
\end{array}\right), \quad\left(\begin{array}{c}
x_{2}(1,1) \\
x_{2}(1,2) \\
x_{2}(2,1) \\
x_{2}(2,2)
\end{array}\right)=\left(\begin{array}{c}
1 \\
1 \\
9 \\
\sqrt[3]{\frac{189 e^{9}+15 e-292,300}{600}}
\end{array}\right) .
$$

\section{Acknowledgements}

The authors gratefully acknowledge the two anonymous reviewers for their careful reading and valuable comments and suggestions.

\section{Funding}

This work is supported by the National Natural Science Foundation of China (Grant No. 11971126) and the Program for Changjiang Scholars and Innovative Research Team in University (Grant No. IRT 16R16).

\section{Availability of data and materials}

Data sharing is not applicable to this article as no data sets were generated or analyzed during the current study.

\section{Competing interests}

The authors declare that they have no competing interests.

\section{Authors' contributions}

All authors contributed equally to this work. All authors read and approved the final manuscript.

\section{Publisher's Note}

Springer Nature remains neutral with regard to jurisdictional claims in published maps and institutional affiliations.

Received: 5 January 2021 Accepted: 21 March 2021 Published online: 01 April 2021

\section{References}

1. Ji, C.: Remarks on the existence of three solutions for the $p(x)$-Laplacian equations. Nonlinear Anal. 74(9), 2908-2915 (2011)

2. Ricceri, B.: A further three critical points theorem. Nonlinear Anal. 71(9), 4151-4157 (2009)

3. Papageorgiou, N.S., Scapellato, A.: Constant sign and nodal solutions for parametric ( $p, 2)$-equations. Adv. Nonlinear Anal. 9(1), 449-478 (2020)

4. Elaydi, S.: An Introduction to Difference Equations. Springer, New York (2005)

5. Agarwal, R.P.: Difference Equations and Inequalities: Theory, Methods and Applications. Dekker, New York (1992) 
6. Yu, J.S., Zheng, B.: Modeling Wolbachia infection in mosquito population via discrete dynamical models. J. Differ. Equ. Appl. 25(11), 1549-1567 (2019)

7. Long, Y.H., Wang, L.: Global dynamics of a delayed two-patch discrete SIR disease model. Commun. Nonlinear Sci. Numer. Simul. 83, 105117 (2020)

8. Stevic, S.: Solvability of a product-type system of difference equations with six parameters. Adv. Nonlinear Anal. 8(1), 29-51 (2019)

9. Guo, Z.M., Yu, J.S.: Existence of periodic and subharmonic solutions for second-order superlinear difference equations. Sci. China Ser. A 46(4), 506-515 (2003)

10. Leng, J.H.: Periodic and subharmonic solutions for $2 n$ th-order $\phi_{c}$-Laplacian difference equations containing both advance and retardation. Indag. Math. 27(4), 902-913 (2016)

11. Liu, X., Zhou, T., Shi, H.P., Long, Y.H., Wen, Z.L.: Periodic solutions with minimal period for fourth-order nonlinear difference equations. Discrete Dyn. Nat. Soc. 2018, 4376156 (2018)

12. Mei, P., Zhou, Z., Lin, G.H.: Periodic and subharmonic solutions for a $2 n$ th-order $\phi_{c}$-Laplacian difference equation containing both advances and retardations. Discrete Contin. Dyn. Syst., Ser. S 12(7), 2085-2095 (2019)

13. Yu, J.S., Shi, H.P., Guo, Z.M.: Homoclinic orbits for nonlinear difference equations containing both advance and retardation. J. Math. Anal. Appl. 352(2), 799-806 (2009)

14. Zhou, Z., Yu, J.S., Chen, Y.M.: Homoclinic solutions in periodic difference equations with saturable nonlinearity. Sci. China Math. 54(1), 83-93 (2011)

15. Zhou, Z., Yu, J.S.: Homoclinic solutions in periodic nonlinear difference equations with superlinear nonlinearity. Acta Math. Sin. Engl. Ser. 29(9), 1809-1822 (2013)

16. Tang, X.H., Chen, J.: Infinitely many homoclinic orbits for a class of discrete Hamiltonian systems. Adv. Differ. Equ. 2013, $242(2013)$

17. Zhou, Z., Ma, D.F.: Multiplicity results of breathers for the discrete nonlinear Schrödinger equations with unbounded potentials. Sci. China Math. 58(4), 781-790 (2015)

18. Tang, X.H.: Non-Nehari manifold method for periodic discrete superlinear Schrödinger equation. Acta Math. Sin. Engl. Ser. 32(4), 463-473 (2016)

19. Lin, G.H., Zhou, Z.: Homoclinic solutions in non-periodic discrete $\phi$-Laplacian equations with mixed nonlinearities Appl. Math. Lett. 64, 15-20 (2017)

20. Lin, G.H., Zhou, Z:: Homoclinic solutions of discrete $\phi$-Laplacian equations with mixed nonlinearities. Commun. Pure Appl. Anal. 17(5), 1723-1747 (2018)

21. Zhang, Q.Q.: Homoclinic orbits for discrete Hamiltonian systems with local super-quadratic conditions. Commun. Pure Appl. Anal. 18(1), 425-434 (2019)

22. Chen, S.T., Tang, X.H., Yu, J.S.: Sign-changing ground state solutions for discrete nonlinear Schrödinger equations. J. Differ. Equ. Appl. 25(2), 202-218 (2019)

23. Lin, G.H., Zhou, Z., Yu, J.S.: Ground state solutions of discrete asymptotically linear Schrödinger equations with bounded and non-periodic potentials. J. Dyn. Differ. Equ. 32(2), 527-555 (2020)

24. Cabada, A., Tersian, S.: Existence of heteroclinic solutions for discrete $p$-Laplacian problems with a parameter. Nonlinear Anal., Real World Appl. 12(4), 2429-2434 (2011)

25. Kuang, J.H., Guo, Z.M.: Heteroclinic solutions for a class of $p$-Laplacian difference equations with a parameter. Appl. Math. Lett. 100, 106034 (2020)

26. Jiang, L.Q., Zhou, Z:: Three solutions to Dirichlet boundary value problems for $p$-Laplacian difference equations. Adv Differ. Equ. 2008, 345916 (2008)

27. Bonanno, G., Candito, P.: Infinitely many solutions for a class of discrete non-linear boundary value problems. Appl. Anal. 88(4), 605-616 (2009)

28. D'Agui, G., Mawhin, J., Sciammetta, A.: Positive solutions for a discrete two point nonlinear boundary value problem with p-Laplacian. J. Math. Anal. Appl. 447(1), 383-397 (2017)

29. Long, Y.H., Chen, J.L.: Existence of multiple solutions to second-order discrete Neumann boundary value problems. Appl. Math. Lett. 83, 7-14 (2018)

30. Zhou, Z., Ling, J.X.: Infinitely many positive solutions for a discrete two point nonlinear boundary value problem with $\phi_{c}$-Laplacian. Appl. Math. Lett. 91, 28-34 (2019)

31. Long, Y.H., Wang, S.H., Chen, J.L.: Multiple solutions of fourth-order difference equations with different boundary conditions. Bound. Value Probl. 2019, 152 (2019)

32. Wang, S.H., Long, Y.H.: Multiple solutions of fourth-order functional difference equation with periodic boundary conditions. Appl. Math. Lett. 104, 106292 (2020)

33. Long, Y.H.: Existence of multiple and sign-changing solutions for a second-order nonlinear functional difference equation with periodic coefficients. J. Differ. Equ. Appl. 26(7), 966-986 (2020)

34. Chen, Y.S., Zhou, Z.: Existence of three solutions for a nonlinear discrete boundary value problem with $\phi_{c}$-Laplacian. Symmetry 12(11), 1839 (2020)

35. Galewski, M., Orpel, A.: On the existence of solutions for discrete elliptic boundary value problems. Appl. Anal. 89(12), 1879-1891 (2010)

36. Ji, J., Yang, B.: Eigenvalue comparisons for boundary value problems of the discrete elliptic equation. Commun. Appl. Anal. 12(2), 189-198 (2008)

37. Heidarkhani, S., Imbesi, M.: Multiple solutions for partial discrete Dirichlet problems depending on a real parameter. J. Differ. Equ. Appl. 21(2), 96-110 (2015)

38. Imbesi, M., Bisci, G.M.: Discrete elliptic Dirichlet problems and nonlinear algebraic systems. Mediterr. J. Math. 13(1), 263-278 (2016)

39. Du, S.J., Zhou, Z:: Multiple solutions for partial discrete Dirichlet problems involving the $p$-Laplacian. Mathematics $8(11), 2030(2020)$

40. Bonanno, G.: A critical points theorem and nonlinear differential problems. J. Glob. Optim. 28(3-4), 249-258 (2004) 